\title{
Los retos en la adaptación al cambio climático en entornos urbanos
}

\author{
Christian Oltra \\ Centro de Investigaciones Energéticas, Medioambientales y Tecnológicas (CIEMAT). \\ Centro de Investigaciones Sociotécnicas \\ christian.oltra@ciemat.es \\ Renato Marín \\ Universitat de Barcelona. Departament de Sociologia i Anàlisi de les Organitzacions \\ renato.mapezz@gmail.com
}

\section{Resumen}

En las próximas décadas, las ciudades de todo el mundo experimentarán los impactos del aumento de la temperatura global. Sin embargo, a diferencia de la mitigación, la adaptación de las ciudades de nuestro entorno permanece como una cuestión secundaria. Este artículo revisa la literatura sobre adaptación al cambio climático en las poblaciones con el objetivo de comprender los retos y las dimensiones implicadas en la adaptación de las ciudades. Identificamos seis grandes retos en la adaptación de las ciudades. Caracterizamos las distintas estrategias de adaptación existentes e ilustramos estas intervenciones a partir de casos concretos documentados en distintas de ellas. Finalmente, discutimos la necesidad de evaluar e investigar de modo sistemático la capacidad de adaptación de las poblaciones de nuestro entorno al cambio climático.

Palabras clave: ciudades; clima; planificación ambiental.

\section{Abstract. Challenges of Adaptation to Climate Change in Urban Environments}

In the coming decades, cities around the world will suffer impacts due to rising global temperatures. However, unlike climate change mitigation, urban adaptation to climate change remains an insignificant issue in our context. This article reviews the broader literature on climate change adaptation with a view to understanding the challenges and dimensions involved in the adaptation of cities to climate change. We identify six main challenges to adaptation. We characterize the existing adaptation strategies and provide examples of documented interventions in cities. Finally, we discuss the need to systematically evaluate and investigate the adaptation of cities to climate change.

Keywords: cities; climate; environmental planning. 


\section{Sumario}

1. Introducción 3. Opciones de adaptación al cambio

2. Los retos en la adaptación al cambio climático en ciudades

climático en entornos urbanos

4. Conclusiones

Referencias bibliográficas

\section{Introducción}

Mitigación y adaptación son los dos pilares de la lucha contra el cambio climático. La mitigación engloba todas aquellas estrategias dirigidas a limitar la intensidad del cambio climático mediante la reducción de las emisiones de gases de efecto invernadero como el $\mathrm{CO}_{2}$. La atmósfera terrestre contiene, en la actualidad, unas $390 \mathrm{ppm}$ (partes por millón) de $\mathrm{CO}_{2}$. Si no se toman medidas de reducción de emisiones, hacia el año 2040, la concentración de $\mathrm{CO}_{2}$ atmosférico podría duplicarse e incluso triplicarse (Houghton et al., 2001; Richardson et al., 2009; página web de Climate Interactive). Esto se traduciría en un incremento de 3 a $4{ }^{\circ} \mathrm{C}$ respecto a los niveles preindustriales. Los impactos derivados de este aumento de temperatura en los sistemas naturales y sociales afectarán a áreas como la salud pública, la agricultura, la disponibilidad de recursos, los ecosistemas y las especies, los bosques y las zonas costeras (Climate Change Science Program, 2003; Comisión Europea, 2009), con costes económicos y sociales significativos (Stern, 2006; Nordhaus, 2007; Pardo, 2007; Giddens, 2010).

En los países en desarrollo, limitar los efectos adversos del cambio climático con actuaciones sobre los sistemas naturales y sociales vulnerables es prioritario (UNFCCC, 2007). Pero el aumento de la temperatura también planteará retos significativos en las áreas urbanas de los países avanzados (Lowe et al., 2009). Las ciudades europeas se verán afectadas, con gran probabilidad, por un incremento en las olas de calor, una disminución de las olas de frío, más episodios de precipitación intensa, una elevación del nivel del mar y la exacerbación de la escasez de agua (Ciscar, 2009; Banco Mundial, 2011). Los impactos en cada ciudad variarán en función de la localización geográfica, así como en función de sus condiciones de vulnerabilidad y capacidad adaptativa. Ciudades como Nueva York, Chicago, Londres, Toronto, Ciudad de México o Ciudad del Cabo han iniciado planes de adaptación al cambio climático (la mayoría de los cuales están disponibles a través de Internet). Las ciudades españolas tampoco están exentas de los riesgos para la salud, la seguridad y el bienestar de los ciudadanos derivados del cambio climático (Baccini et al., 2008). Esto obliga a reflexionar sobre las amenazas climáticas futuras, así como sobre las respuestas de adaptación más adecuadas.

La adaptación al cambio climático se define como el conjunto de estrategias e intervenciones en distintos ámbitos destinadas a reducir la vulnerabilidad de los sistemas naturales (bosques, tierras de cultivo, zonas costeras, etc.) y 
humanos (ciudades, sectores económicos, sociedades) frente a los efectos reales o esperados del cambio climático inevitable (Smit y Wandel, 2006; Glick et al., 2009). Aunque la literatura en adaptación al cambio climático ha crecido significativamente desde 2005 (Arnell, 2010; Berrang-Ford et al., 2011), esta ha recibido tradicionalmente una menor atención que la mitigación por parte de las comunidades políticas y científicas (Füssel y Klein, 2006; Bierbaum, 2008). La mitigación es una cuestión delimitada, mientras que la adaptación tiene un carácter más difuso, requiere de modificaciones en sistemas humanos y sociales en diferentes escalas y suele ser, solo en parte, una respuesta a los elementos climáticos (Berrang-Ford et al., 2011). La adaptación de las ciudades al cambio climático plantea retos muy significativos que deben ser analizados de modo sistemático. En alguna medida, la planificación para el cambio climático se configura como un nuevo problema para la planificación urbana (Pizarro, 2009).

Este artículo trata de dar respuesta a dos cuestiones básicas: ¿Cuáles son los retos fundamentales de adaptación al cambio climático en los entornos urbanos? y ¿̇de qué estrategias de adaptación disponemos en las ciudades para hacer frente al cambio climático? El artículo se basa en una revisión de la literatura sobre adaptación al cambio climático en entornos urbanos. Se han consultado artículos académicos sobre adaptación publicados en los últimos diez años, así como informes y documentos generados por administraciones y organizaciones locales, nacionales e internacionales, dado que una parte de las intervenciones de adaptación están documentadas fuera de la literatura científica (Berrang-Ford et al., 2011). Se ha prestado una especial atención a las guías de adaptación elaboradas por administraciones locales de ciudades de países avanzados en los últimos cinco años.

En la siguiente sección, se revisan los retos fundamentales por lo que se refiere a la adaptación en las ciudades. En la sección tercera, se consideran las principales estrategias de adaptación al cambio climático en contextos urbanos y se ilustra el proceso de adopción de las mismas a partir de ejemplos concretos. Finalmente, se establecen algunas conclusiones y se discuten las implicaciones más relevantes.

\section{Los retos en la adaptación al cambio climático en entornos urbanos}

En la última década, el interés y la aceptación de la necesidad de adaptación al cambio climático ha crecido significativamente en las comunidades políticas (Glick et al., 2009). De la misma manera lo ha hecho la literatura científica al respecto (Heller y Zavaleta, 2009; Glick et al., 2009). La necesidad de combinar acciones urgentes de adaptación y mitigación ha sido recogida en la Convención Marco de Cambio Climático de las Naciones Unidas (UNFCCC). Asimismo, se han establecido mecanismos específicos para la financiación de proyectos de adaptación en países vulnerables, como el Adaptation Fund, dados los impactos ya constatables del cambio climático en algunos países. Sin embargo, persiste un déficit de atención a las cuestiones relacionadas con la 
adaptación (Burton, 2005; Bierbaum, 2008). La tendencia en las comunidades políticas y científicas a destinar recursos a la mitigación ha producido una falta de comprensión de los retos, las necesidades y las soluciones asociadas a la adaptación al cambio climático (Burton et al., 2006).

Las ciudades difieren en sus niveles de exposición y sensibilidad a los impactos del cambio climático. Ello es debido, en primer lugar, a su localización geográfica (Banco Mundial, 2011). Ciudades situadas en zonas costeras de baja elevación en el este de Asia pueden verse afectadas por la subida del nivel del mar y el incremento de las precipitaciones (Satterthwaite et al., 2007; Banco Mundial, 2008, 2011; International Housing Coalition, 2011). En el Mediterráneo, las ciudades deberán hacer frente, con una alta probabilidad, a temperaturas más elevadas, una mayor frecuencia de noches tropicales, una disminución de las precipitaciones y un aumento de las sequías (Giannakopoulos et al., 2005). En el sur de Europa, el incremento de la temperatura podría traducirse en un aumento de la mortalidad, así como en una disminución de la calidad de vida de los habitantes de las ciudades (Ballester, 2005). Las ciudades del norte de Europa podrían beneficiarse de una menor incidencia de olas de frío (Ciscar, 2009; Lomborg, 2008), pero sufrir las consecuencias de la subida del nivel del mar o la disminución de la disponibilidad de agua (Schernewski et al., 2011).

A grandes rasgos, todas las ciudades harán frente a algún tipo de impacto climático más o menos severo (los más importantes han sido recogidos en la tabla 1). En primer lugar, un clima más cálido y una mayor frecuencia de olas de calor se traducirán en una mayor mortalidad y morbilidad (Kovats y Hajat, 2008). Los episodios de calor extremo representan una amenaza seria para las poblaciones de las ciudades, así como un reto para la salud pública. Un episodio significativo fue la ola de calor de agosto de 2003, que causó algo más de 15.000 muertes en veinte días en Francia (Fouillet et al., 2006) y entre 6.500 y 8.600 en España (Simon et al., 2005). La ciudad de París registró los excesos en la mortalidad más altos de toda Francia (Fouillet et al., 2006). Y es muy probable que se incremente la frecuencia de olas de calor en el centro de Europa y Europa del sur en el futuro (Ciscar, 2009), con especial incidencia en las ciudades (Fischer et al., 2012). Otros posibles impactos en las ciudades europeas incluyen posibles inundaciones por la subida del nivel del mar (más probable en el centro, el norte y el sur de Europa) e inundaciones por un aumento del cauce de los ríos (más probable en el centro de Europa). El cambio climático podría también producir cambios en las enfermedades, así como un incremento en el riesgo de accidentes y pérdidas de bienestar por eventos extremos como tormentas y riadas (Ciscar, 2009; McMichael et al., 2006). Todos estos impactos tendrán repercusiones en aspectos diversos como las infraestructuras, la salud pública, el bienestar de la población o la gestión de recursos.

Los impactos del cambio climático pueden acrecentar, también, otros problemas urbanos socioambientales. El incremento en la temperatura interactúa con estresores urbanos como la contaminación del aire (Rosenzweig et al., 


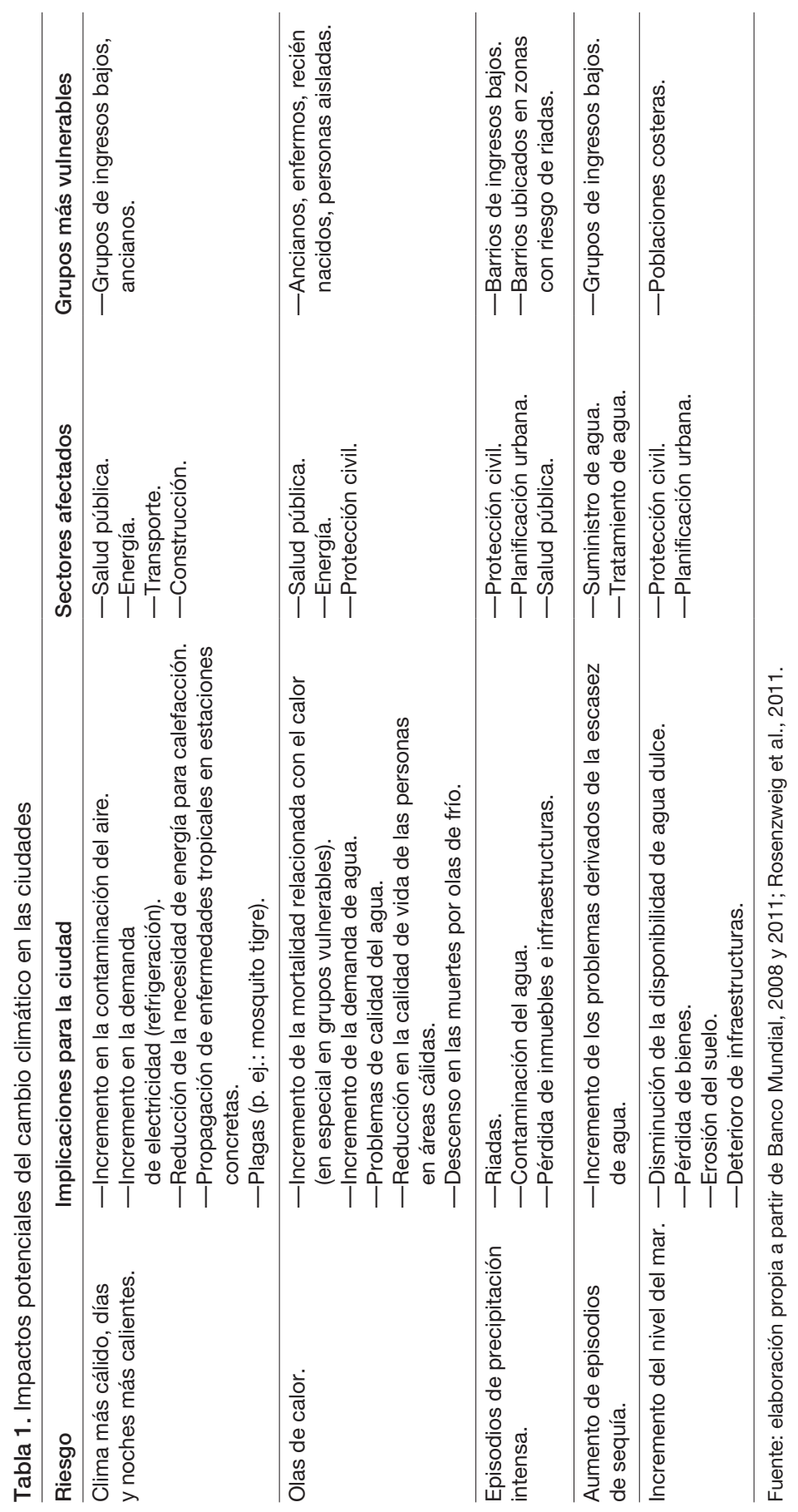


2011), lo cual genera una sinergia negativa para el bienestar de los ciudadanos. El aumento de las temperaturas puede agravar el impacto de la contaminación atmosférica urbana sobre la salud de la población, en especial en el caso de compuestos como el ozono (Ballester, 2005). Asimismo, un clima más cálido se puede traducir en un incremento del consumo de electricidad y la demanda de agua en los meses de verano. Esta interacción puede plantear nuevos retos en la gestión urbana (Rosenzweig et al., 2011), al acrecentar algunos problemas de escasez y suministro ya existentes y requerir una coordinación e integración de estrategias en distintos sectores.

De la misma manera que las ciudades difieren en su vulnerabilidad y capacidad de adaptación frente a los episodios climáticos, en el interior de las ciudades, los grupos sociales y las poblaciones difieren en su vulnerabilidad. El estudio clásico de Klinenberg sobre la ciudad de Chicago (2002) evidencia la vulnerabilidad de algunos segmentos sociales en ciudades avanzadas ante los impactos del calor extremo. La ola de calor acaecida en julio de 1995 causó cientos de muertos entre la población más vulnerable, y ello puso de manifiesto las dificultades organizativas de las ciudades para hacer frente a eventos climáticos extremos (Semenza et al., 1996). Las muertes se repartieron de modo desigual. Las condiciones de vulnerabilidad más significativas fueron: vivir solo, no abandonar el hogar diariamente, falta de acceso a un medio de transporte, estar enfermo, no tener contactos sociales cerca y no disponer de aire acondicionado (Semenza et al., 1996; Klinenberg, 2002). Esto ejemplifica el efecto de factores sociales como el aislamiento en la vulnerabilidad individual frente a los impactos climáticos.

Para hacer frente a los impactos derivados del cambio climático inevitable, es necesario el desarrollo de intervenciones de adaptación efectivas. La adaptación al cambio climático se configura como un nuevo problema de planificación urbana, dada la necesidad de considerar la diversidad de sinergias, conflictos y equilibrios entre estrategias de mitigación y de adaptación, así como entre las estrategias de adaptación y las cuestiones de planificación local y desarrollo urbano más generales (Biesbroek et al., 2009; Laukkonen et al., 2009; Pizarro, 2009), La adaptación es un fenómeno específico, dado que cada ciudad presenta unas condiciones únicas de exposición, sensibilidad y capacidad adaptativa (Banco Mundial, 2011; Rosenzweig et al., 2011), pero los retos de la adaptación son comunes. Desde las estrategias más sencillas, como incrementar el número de tejados blancos en una población, hasta estrategias más complejas que impliquen cambios en el diseño o en las infraestructuras de las ciudades, todas conllevan ciertos retos en alguno de los seis factores sistematizados a continuación:

- Políticas públicas y planificación urbana. La adaptación de las ciudades al cambio climático requiere del diseño, del desarrollo y de la implementación de estrategias, programas y actividades que preparen a las ciudades de modo efectivo para los posibles impactos climáticos. Investigaciones como las de Biesbroek et al. (2009) consideran que la adaptación al cambio climático altera los límites 
y las estructuras administrativas tradicionales en las que opera la planificación urbana. La adaptación plantea nuevos retos para la planificación urbana, en un escenario de problemas de coordinación entre el ámbito público y el privado, retos de gobierno y financiación, crecimiento de las ciudades y cambios en la estructura urbana, así como necesidad de mitigación del cambio climático (Biesbroek et al., 2009; Clarck, 2009). Biesbroek et al. (2009) identifican tres factores que dificultan la búsqueda de sinergias entre mitigación y adaptación en la política y la planificación urbanas: las diferencias en el horizonte temporal (largo plazo en la mitigación frente a corto plazo necesario en la adaptación); las diferencias en las escalas administrativas implicadas (la adaptación es, en esencia, local), y las diferencias en partes interesadas que están implicadas. La adaptación al cambio climático se añade, en alguna medida, a los problemas y a los retos ya existentes en la política urbana.

Los gobiernos y las organizaciones públicas locales tienen un papel fundamental en la adaptación al cambio climático (Lowe et al., 2009). Para ello, deben hacer frente a retos diversos, como: incrementar el compromiso político con la adaptación al cambio climático; diseñar intervenciones efectivas de adaptación (estructurales, comportamentales, tecnológicas, etc.); fomentar la integración de estas intervenciones en otras políticas urbanas; destinar recursos para su implementación, e incrementar la coordinación y la implicación de los actores locales y las comunidades en su correcta implementación (Rojas Blanco, 2006; Sánchez-Rodríguez, 2009; Eriksen et al., 2012; Rosenzweig et al., 2011).

La revisión de distintos casos de estudio (Lowe et al., 2009) muestra que hay diversos elementos que definen las estrategias urbanas de adaptación de éxito: un liderazgo fuerte por parte del alcalde u otro responsable político; la incorporación de conocimiento científico a través de universidades locales; la implicación de las distintas partes interesadas; la sinergia con las estrategias de mitigación del cambio climático por parte de los gobiernos locales, y una búsqueda creativa de apoyo y financiación. En este sentido, en la medida en que los gobiernos locales sean capaces de evaluar la capacidad de sus comunidades para hacer frente al cambio climático, así como de determinar las respuestas más apropiadas, serán factores determinantes en la resiliencia de las ciudades frente al cambio climático (Laukkonen et al., 2009).

- Recursos. La adaptación al cambio climático conllevará costes significativos, pero también beneficios derivados de los impactos evitados y las oportunidades generadas (Callaway, 2004; Adger et al., 2005). Las estrategias de adaptación requerirán la movilización de recursos económicos y sociales públicos y privados escasos. Pues, si bien los individuos, las organizaciones y los gobiernos llevan a cabo acciones de adaptación de modo espontáneo, en respuesta a los cambios en el clima (Callaway, 2004), e indirecto, numerosas acciones de adaptación requerirán decisiones de inversión con cierto riesgo. Por este motivo, la efectividad y la eficiencia económica son criterios fundamentales en la evaluación de las estrategias de adaptación (Adger et al., 2005). Conocer 
los costes reales en recursos de implementar una opción de adaptación es fundamental en la toma de decisiones de individuos y gobiernos.

En el caso de decisiones de adaptación proactivas, es decir, previas a la detección o materialización del impacto, la movilización de recursos para la adaptación dependerá, en alguna medida, de un cálculo de los daños previstos por el impacto climático, el beneficio neto de la adaptación y los daños impuestos o no evitables por la adaptación (Callaway, 2004). Pero es poco probable que únicamente el cálculo del beneficio neto de la adaptación determine la toma de decisiones en cuestiones de adaptación. Las decisiones de adaptación se verán influidas por los valores y las actitudes de los actores en torno a la adaptación y al desarrollo socioeconómico (Haddad, 2005; O’Brien, 2009), las prácticas y rutinas de individuos, organizaciones y gobiernos, así como las estructuras de gobernanza y colaboración (Dietz et al., 2003). La estimación de los costes y de los beneficios sociales y económicos de la adaptación será, asimismo, aunque fundamental, problemática (Adger et al., 2005).

- Eficacia colectiva. Algunas características de las comunidades y de las poblaciones, como la eficacia colectiva o el capital social, pueden incidir en el desarrollo de las estrategias de adaptación al cambio climático (Ebi y Semenza, 2008; Eriksen et al., 2012). Como en ámbitos como la lucha contra la delincuencia o la promoción de la salud (Ahern y Galea, 2011), la habilidad de individuos y organizaciones para trabajar de modo conjunto puede influir en la capacidad de desarrollar estrategias eficientes de adaptación al cambio climático. Una mayor implicación pública y una coordinación entre actores pueden favorecer el diseño, la implementación y el seguimiento de las estrategias de adaptación (Lowe et al., 2009). Asimismo, las organizaciones locales pueden desempeñar un papel fundamental en el desarrollo de estrategias de adaptación locales, como se ha puesto de manifiesto en estudios realizados en países de ingresos bajos (Rojas Blanco, 2006). En este sentido, las estrategias de adaptación locales basadas en la comunidad (Ebi y Semenza, 2008) persiguen ayudar a las sociedades a estar más preparadas frente a los riesgos para la salud y el bienestar derivados del cambio climático.

- Tecnología e infraestructura. Por lo general, se considera que el desarrollo de las estrategias de adaptación es posible con las tecnologías disponibles en la actualidad. Gran parte de las tecnologías ya utilizadas para reducir la vulnerabilidad frente a eventos climáticos se podrían trasladar al ámbito de la adaptación (Machado, 2009), pero las implicaciones tecnológicas de la adaptación varían con las distintas opciones de adaptación. Algunas intervenciones pueden requerir modificaciones infraestructurales complejas y caras, como la gestión de la escasez del agua o la construcción de diques y la reubicación y protección de edificios para hacer frente a la subida del nivel del mar (Banco Mundial, 2011). Otras intervenciones requerirán modificaciones más sencillas, como las destinadas a reducir el efecto isla de calor en las ciudades a través del incremento de la superficie arbolada o la modificación de los techos de los 
edificios. Pero, en términos generales, los retos tecnológicos son menores a los existentes en la mitigación del cambio climático. El reto material en la adaptación no reside tanto en la innovación tecnológica como en la correcta elección de las soluciones, así como en la evaluación de sus posibles consecuencias no buscadas, su legitimidad y equidad (Adger et al., 2005).

- Información y conocimiento. Como en otros ámbitos de la gobernanza ambiental, la existencia de información adecuada sobre los sistemas naturales, así como sobre la interacción de estos con los sistemas humanos y sociales, es fundamental (Dietz et al., 2003). El conocimiento empírico sobre los impactos del cambio climático, la exposición y vulnerabilidad de los sistemas urbanos y la efectividad y los posibles impactos de las medidas de adaptación al cambio climático constituyen la base para el diseño de estrategias de adaptación efectivas (Füssel y Klein, 2006; Smit y Wandel, 2006). Los retos en el conocimiento científico de estas cuestiones son significativos, dada la diversidad de sistemas y niveles implicados. Pero, asimismo, la incorporación de este conocimiento en la toma de decisiones y la consideración de la incertidumbre podría plantear retos en la adaptación al cambio climático (Berrang-Ford et al., 2011; National Oceanographic and Atmospheric Administration, 2011), aunque la existencia de más investigación no se traduce, necesariamente, en intervenciones políticas efectivas.

Por un lado, existen incertidumbres irreductibles en la ciencia del clima. Por otro, el reto fundamental reside, tal vez, en tomar mejores decisiones, y no solo en hacer mejores predicciones (Olsen, 2011). Los responsables políticos necesitan información sobre los tipos y la magnitud de esta incertidumbre, así como sobre la naturaleza y el grado de ignorancia científica y desacuerdo (Dietz et al., 2003). Necesitan, también, información que sea accesible, a partir de la cual se puedan tomar decisiones (Lowe et al., 2009). Asimismo, es fundamental la adecuación de los datos a la escala propia de cada población, dado el carácter local de la adaptación (Lowe et al., 2009). Finalmente, es necesario analizar los valores sociales que dirigen las distintas opciones de adaptación, así como los posibles efectos negativos no previstos de las mismas sobre las comunidades, las sociedades y los ecosistemas (Eriksen et al., 2012).

- Actitudes, conciencia y motivación. La existencia de un conocimiento preciso sobre los futuros impactos climáticos en nuestras ciudades no garantiza la implementación de estrategias y mecanismos que favorezcan la adaptación. Las percepciones, las normas sociales y las motivaciones de individuos, organizaciones y gobiernos (Haddad, 2005) desempeñan un papel fundamental en el desarrollo de adaptaciones al cambio climático. Pocos estudios han abordado los elementos influyentes en la adopción, por parte de individuos y organizaciones, de acciones de adaptación frente al cambio climático. Pero una amplia línea de investigación se ha desarrollado en el ámbito de la preparación frente a desastres naturales como inundaciones o huracanes (Tierney et al., 2001). 
Como en el ámbito de la adopción de innovaciones, el comportamiento en salud (Abroms y Maibach, 2008) o el comportamiento de ahorro energético (Wilson y Dowlatabadi, 2007), la adopción, por parte de los individuos, de estrategias de prevención frente a los desastres depende de diversos factores, entre ellos, factores psicológicos relacionados con la ansiedad, la eficacia personal y la confianza y los elementos relacionados con la consideración de costes y beneficios (Bauman y Sims, 1978), así como elementos sociales y del entorno. El conocimiento sobre la posibilidad de un impacto climático futuro puede influir en la adopción de estrategias de adaptación, pero no es un factor determinante en la adopción (Bauman y Sims, 1978). Así que, aunque la conciencia sobre el cambio climático se considera un elemento fundamental en la difusión de estrategias de adaptación (Rosenzweig et al., 2011), es poco probable que la mera transmisión de información al respecto logre cambios conductuales significativos.

\section{Opciones de adaptación al cambio climático en ciudades}

Considerados los retos derivados del cambio climático a los que, con gran probabilidad, deberán hacer frente las ciudades en los próximos años, una cuestión fundamental es analizar las estrategias de adaptación que se han llevado a cabo en las ciudades para hacer frente a estos retos. En los últimos años, se ha desarrollado una cantidad significativa de informes y de guías especializadas de gobiernos locales y organismos internacionales sobre las intervenciones básicas de adaptación al cambio climático en entornos urbanos (Lowe et al., 2009; Banco Mundial, 2008). Aunque los retos difieren de modo significativo en función de la localización geográfica de las ciudades, su vulnerabilidad y su capacidad adaptativa, es posible distinguir cuatro tipos fundamentales de opciones y estrategias de adaptación:

\section{Destinadas a la gestión del calor}

El incremento previsible de las temperaturas y de los episodios de olas de calor en las ciudades del sur de Europa y otras latitudes plantea dos retos fundamentales: la mitigación del efecto isla de calor y la preparación de las comunidades frente a las olas de calor. El efecto isla de calor, por el que las áreas urbanas alcanzan en verano temperaturas superiores a las áreas circundantes, es un fenómeno ampliamente estudiado (Oke, 1982). El clima de las ciudades depende de una gran variedad de factores naturales y humanos interconectados, pero existen ciertas intervenciones que se consideran efectivas en la reducción del efecto isla de calor (Foster et al., 2011; Environmental Protection Agency, 2012):

a) El incremento de la vegetación y el arbolado.

b) La sustitución de techos y azoteas por techos fríos (ajardinados o con mayor reflectancia solar).

c) El incremento en el uso de pavimentos fríos. 
Existe bastante evidencia sobre el efecto positivo local de estas tres intervenciones: temperaturas menos elevadas en verano, menor uso de energía para refrigeración, menores niveles de contaminación urbana, menores niveles de ruido urbano y mejora del bienestar de las poblaciones locales (Akbari y Rose, 2001; Foster et al., 2011; Environmental Protection Agency, 2012).

Incrementar la reflectancia urbana a partir de techos y pavimentos fríos puede reducir la temperatura urbana en verano y mejorar la calidad del aire de las ciudades (Akbari y Rose, 2001), pero, además, puede suponer una reducción en las emisiones urbanas de $\mathrm{CO}_{2}$, debido al menor uso de energía (Akbari et al., 2009). Aunque el impacto sobre las emisiones globales de $\mathrm{CO}_{2}$ de intervenciones como los techos blancos ha sido discutido en algunas investigaciones (Jacobson y Ten Hoeve, 2012), esta intervención ha sido aplicada en numerosas ciudades de los Estados Unidos. Los techos fríos permiten reducir la temperatura máxima del edificio en verano en $10^{\circ} \mathrm{C}$. Ciudades como Nueva York han desarrollado, incluso, programas de voluntariado para convertir las azoteas de edificios públicos y de viviendas en techos fríos, a través de una capa de pintura blanca reflectante. Iniciativas similares se desarrollan en otros países ${ }^{1}$.

Desde 2003, se han implementado, también, en distintos países de Europa, medidas de salud pública para hacer frente a las olas de calor (Kovats y Hajat, 2008). El incremento en la morbilidad y en la mortalidad debidas a olas de calor es una de las amenazas fundamentales en los entornos urbanos derivadas del cambio climático (Kovats y Hajat, 2008; Rosenzweig et al., 2011; McMichael y Butler, 2009). La preparación de las comunidades ha implicado estrategias en distintos ámbitos, dirigidas a: mejorar la respuesta del sistema de salud (formación y capacidad de respuesta); incrementar la conciencia y la educación pública al respecto, en especial de grupos vulnerables (ancianos, enfermos, grupos de estatus socioeconómico bajo, minusválidos); aumentar el capital social de las comunidades; mejorar los hogares (introducción de aparatos de aire acondicionado), o habilitar instalaciones adaptadas en las ciudades, como la apertura de centros cívicos, centros de la tercera edad y bibliotecas refrigerados (denominados cooling centers en Estados Unidos). Sin embargo, existe una evidencia limitada respecto a la efectividad de estas estrategias para prevenir la mortalidad relacionada con el calor en entornos comunitarios (Kovats y Hajat, 2008).

\section{Destinadas a la gestión de recursos}

La adaptación de las ciudades frente al cambio climático requerirá una gestión más eficiente de recursos como el agua, la energía o los alimentos. La gestión del agua será especialmente relevante en poblaciones afectadas por sequías (Banco Mundial, 2011). Numerosas ciudades tienen una larga tradición de gestión de la escasez de agua, pero los impactos del cambio climático podrían requerir medidas adicionales de adaptación, en especial en los países pobres (Satterthwaite et al., 2007; Banco Mundial, 2008; International Housing

1. Véase la página web de $N Y C^{\circ}$ Cool Roofs, así como la iniciativa EU Cool Roofs Council. 
Coalition, 2011). Las opciones de adaptación incluyen desde la mejora de los sistemas de almacenamiento de agua hasta la mejora en la recogida del agua de lluvia, la reutilización hídrica, la desalinización o la mejora en la eficiencia del uso del agua (Banco Mundial, 2008). Un buen ejemplo documentado de gestión integral hídrica y de los riesgos de riadas lo constituye el programa del condado de King, Washington (Lowe et al., 2009).

La gestión de la energía también se verá afectada por los impactos previsibles del cambio climático. Un aumento de la demanda de electricidad, así como amenazas a la fiabilidad de la red y las infraestructuras energéticas son los impactos principales (SPUR, 2011). Aunque se asume que los sistemas energéticos de las ciudades en países avanzados están diseñados para hacer frente a la variabilidad climática, el aumento de la temperatura urbana y la mayor incidencia de eventos climáticos extremos podría afectar a la capacidad del sistema eléctrico de cubrir las necesidades de demanda de electricidad (Rosenzweig et al., 2011). Reducir la dependencia de una sola fuente de energía a través de las energías renovables, incrementar la fortaleza de los sistemas de transmisión y distribución e incrementar la eficiencia energética de las ciudades se consideran las opciones de adaptación más adecuadas (Banco Mundial, 2008; Hamin y Gurran, 2009). Aunque algunos de los retos en el ámbito energético son específicos de la adaptación a los impactos del cambio climático (diversificación en las fuentes y fortalecimiento del sistema de suministro), en buena medida coinciden con los planes de mitigación del cambio climático elaborados desde distintos ámbitos (Rifkin, 2011; Lerch, 2007).

\section{Destinadas a la gestión de catástrofes}

Un incremento en los episodios de tormentas y riadas junto con el aumento del nivel del mar puede plantear riesgos significativos para las poblaciones y las propiedades urbanas (Rosenzweig et al., 2011). Las ciudades en los países avanzados son, previsiblemente, menos vulnerables a estos eventos climáticos. Pero episodios como la subida del nivel del mar podrían plantear pérdidas económicas significativas incluso en ciudades como Nueva York (Rosenzweig et al., 2011). En los países pobres, el riesgo se incrementa por la posible incapacidad de las infraestructuras para hacer frente a estos riesgos en sistemas urbanos con una población creciente y deficiencias estructurales (Satterthwaite et al., 2007; Banco Mundial, 2011; International Housing Coalition, 2011). Distintas organizaciones han desarrollado guías de actuación dirigidas a orientar a los responsables políticos de los países en desarrollo sobre la gestión del riesgo por riadas, tormentas e inundación de las costas (World Metereological Organization, 2008; Abhas et al., 2012). Entre las opciones de adaptación disponibles, destacan la construcción de diques, la reubicación de edificios y áreas agrícolas o la creación de humedales (Banco Mundial, 2011; Rosenzweig et al., 2011). Todas ellas pueden implicar ciertos impactos no intencionados sobre otras poblaciones o áreas de la ciudad, que deberían ser considerados en su planificación (Rosenzweig et al., 2011). 


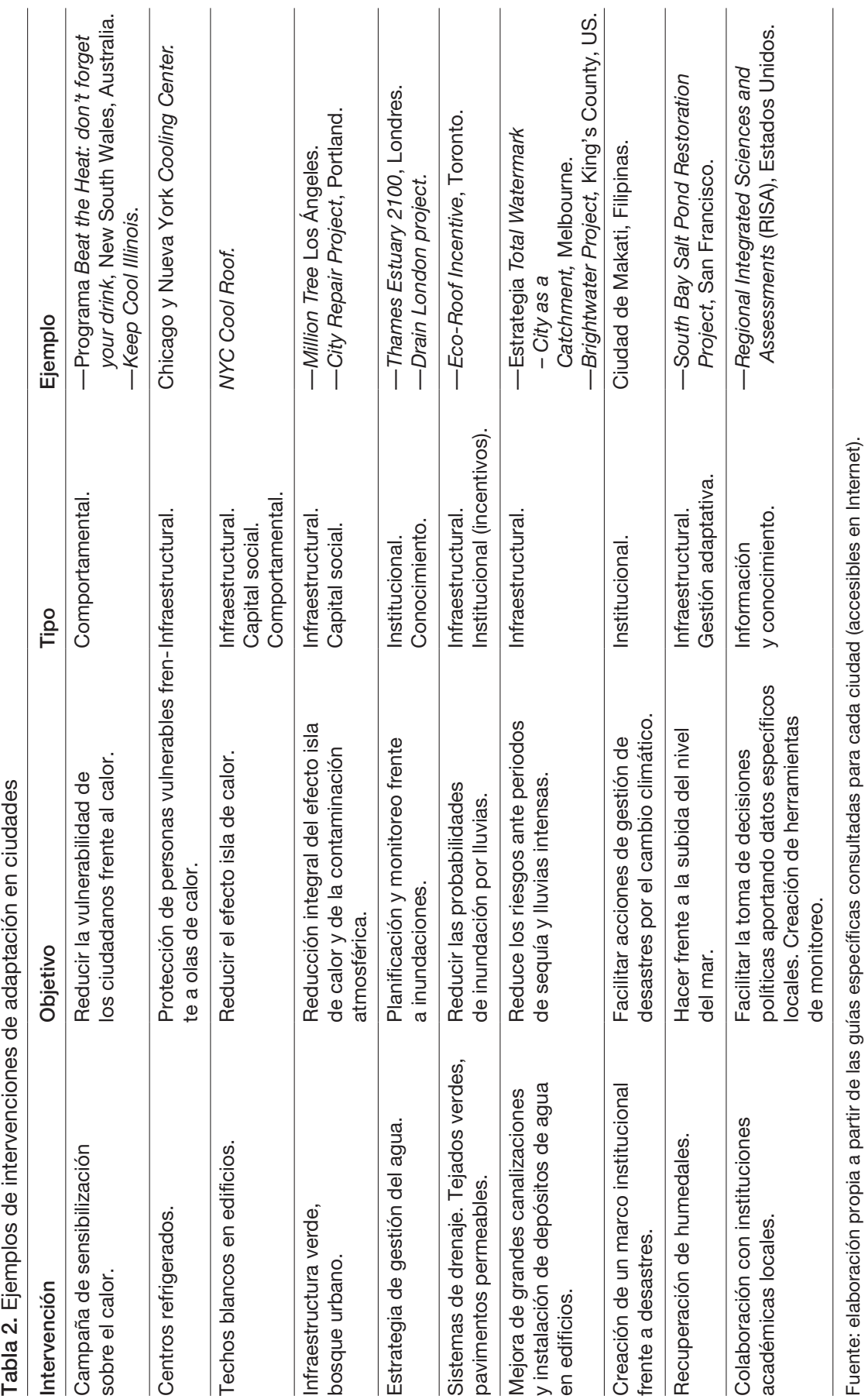




\section{Estrategias indirectas}

Es posible favorecer la adaptación al cambio climático al mismo tiempo que reducir las emisiones de gases de efecto invernadero. La sinergia entre mitigación y adaptación ha sido analizada en ámbitos como la agricultura (Smith y Olesen, 2010). Aunque en el ámbito urbano esta cuestión ha sido menos explorada, es esperable que gran parte de las estrategias destinadas a la reducción de las emisiones de $\mathrm{CO}_{2}$ en las ciudades pueda favorecer, también, la adaptación frente al cambio climático. Por ejemplo, la difusión de infraestructuras verdes (árboles, vegetación, parques, tejados verdes) disminuye la emisión de $\mathrm{CO}_{2}$ en las ciudades, al tiempo que reduce la contaminación atmosférica, mejora la calidad de vida de los ciudadanos y disminuye el efecto isla de calor (Foster et al., 2011). Otras intervenciones destinadas a la reducción de la contaminación urbana, el ahorro de agua o el desarrollo socioeconómico y la lucha contra la pobreza urbana también pueden tener un impacto significativo futuro en la adaptación al cambio climático; por ejemplo: la mejora general en la atención sociosanitaria puede optimizar la respuesta de una ciudad frente a episodios de olas de calor (McMichael y Butler, 2009). Asimismo, el desarrollo socioeconómico puede disminuir la vulnerabilidad de la población local a los efectos inesperados del clima.

En la tabla 2, se puede observar la diversidad de intervenciones de adaptación al cambio climático que han sido puestas en marcha en ciudades de todo el mundo. Gran parte de las intervenciones se han producido en ciudades norteamericanas, donde más intervenciones de adaptación han sido documentadas (Berrang-Ford et al., 2011). Algunas de estas intervenciones tienen objetivos específicos, con beneficios esperados en un único sector. Otras, por el contrario, conjugan modificaciones técnicas con intervenciones sociales y urbanas dirigidas a producir cambios integrales que mejoren la actuación medioambiental de la ciudad, al tiempo que incrementan la capacidad adaptativa de la misma. Un ejemplo lo constituye el programa Million Tree, de la ciudad de Los Ángeles ${ }^{2}$. Se trata de un esfuerzo comunitario dirigido a incrementar el bosque urbano de la ciudad con especies locales, tanto en espacios públicos como en propiedades privadas, con el fin de reducir el efecto isla de calor. Plantar árboles en propiedades privadas provee sombra y reduce, asimismo, las necesidades de refrigeración. También puede contribuir a la mejora del drenaje del agua de lluvia.

Estrategias como Million Tree o City Repair Project permiten avanzar en la adaptación al cambio climático sin comprometer recursos que pudieran ser destinados a acciones de mitigación. Están basadas en una implementación local a partir del esfuerzo de agentes sociales (organizaciones locales, empresas y ciudadanos) que, de forma voluntaria, pueden ceder espacio para la plantación de árboles, hacer donaciones o participar en las tareas de plantación y mantenimiento de los árboles. De este modo, estas intervenciones conllevan

2. http://www.milliontreesla.org/. 
beneficios en términos de reducción del efecto isla de calor, pero también de generación de capital social, en tanto que fomenta la corresponsabilidad de los agentes sociales y de concienciación. Los factores que hacen de este programa una estrategia de éxito son, entre otros, la conjugación de esfuerzos públicos y privados, la naturaleza múltiple de sus beneficios y la simpleza técnica de la intervención.

\section{Conclusiones}

La adaptación de las poblaciones al cambio climático plantea nuevos retos para la planificación urbana y la gestión pública de las ciudades que se añaden a otros retos derivados del gobierno y la financiación local, la coordinación entre la gestión pública y la privada, los procesos macrosociales, como el crecimiento urbano o los cambios en la estructura urbana, y la mitigación del cambio climático (Biesbroek et al., 2009; Clarck, 2009). Los riesgos del cambio climático no han sido incorporados en las decisiones sobre el desarrollo socioeconómico urbano, como tampoco lo ha sido la conciencia sobre la necesidad de la adaptación (Burton, 2005). La adaptación al cambio climático ha recibido una menor atención en las políticas nacionales y urbanas que la mitigación (Burton, 2005), pero el carácter inevitable del cambio climático y sus impactos previsibles sobre las ciudades hacen necesario el análisis sistemático de la adaptación al cambio climático en entornos urbanos.

El objetivo de este artículo ha sido la discusión y la sistematización, a partir de la revisión de la literatura existente, de los retos principales que la adaptación al cambio climático plantea para las ciudades de nuestro entorno, así como de las respuestas posibles de adaptación. De la revisión, se derivan dos ideas básicas en el ámbito de la planificación y el diseño urbanos. En primer lugar, la adaptación de las ciudades al cambio climático requiere de una correcta evaluación de las necesidades locales de adaptación, del diseño de intervenciones de adaptación efectivas y sostenibles y de una adecuada implementación de estas intervenciones, que prepare de modo efectivo a las ciudades para los posibles impactos climáticos. En segundo lugar, las estrategias de adaptación están, en gran medida, disponibles, y el éxito de la adaptación al cambio climático no depende en exclusiva de la magnitud del riesgo climático ni de la disponibilidad de opciones de adaptación, sino de la capacidad de implementar estas opciones (Füssel y Klein, 2006).

La adaptación al cambio climático altera los límites y las estructuras administrativas tradicionales en las que opera la planificación urbana (Pizarro, 2009). En este sentido, es previsible que los gobiernos locales y las organizaciones de las ciudades de nuestro entorno tengan serias dificultades para implementar intervenciones de adaptación efectivas. ¿Seremos capaces de preparar nuestras ciudades para un clima más cálido? Desde nuestro punto de vista, numerosos factores influirán en el resultado. Entre ellos, el liderazgo político, la incorporación eficiente de conocimiento científico y local, la implicación de las distintas partes interesadas (expertos, profesionales de todos los sectores 
afectados, etc.) en la búsqueda de soluciones, la generación de sinergias con las estrategias de mitigación del cambio climático o la búsqueda creativa de apoyo y recursos.

Un reto de particular importancia será la necesidad de buscar equilibrios y sinergias entre las medidas de mitigación (reducción de emisiones de gases de efecto invernadero) y las medidas de adaptación. En la actualidad, sabemos que es poco probable que una acción de adaptación se produzca motivada únicamente por el cambio climático (Smit y Wandel, 2006). Los éxitos documentados de adaptación se han producido cuando las medidas se han incorporado en los planes más amplios de gestión del riesgo, planificación del suelo, gestión de recursos, desarrollo, etc. (Smit y Wandel, 2006), lo que hace imprescindible la búsqueda de sinergias entre la adaptación al cambio climático y otras intervenciones de mitigación o reforma ambiental. Sin embargo, hay evidencias de que las acciones de adaptación pueden entrar en contradicción con las acciones de mitigación, así como con otras intervenciones urbanas, como las destinadas a incrementar la densidad urbana (Hamin y Gurran, 2009).

La adaptación al cambio climático también plantea numerosos retos para la investigación en ciencias sociales. Una idea fundamental es que la capacidad adaptativa de una ciudad está en función de numerosos determinantes no climáticos, tales como los recursos económicos, la tecnología, la información, la infraestructura, las instituciones, las habilidades o el grado de desigualdad; aspectos todos ellos que requieren ser investigados (Yohe y Tol, 2002). En nuestra revisión, hemos dejado de lado muchas cuestiones objeto de estudio de la sociología, la economía o la geografía urbana que pueden tener una influencia significativa en el desarrollo y en la efectividad de las intervenciones de adaptación, pero somos conscientes de la necesidad de realizar investigación empírica de todas estas cuestiones.

Sin ánimo de ser exhaustivos, algunas cuestiones objeto posible de la investigación futura son la evaluación de la vulnerabilidad y las necesidades de adaptación de las ciudades frente al cambio climático; el estudio de los factores geográficos, sociopolíticos y económicos urbanos y su papel en el desarrollo y en el éxito de las intervenciones de adaptación; el estudio de la efectividad de los distintos mecanismos institucionales (de mercado, nudges, regulatorios, burocráticos, de márketing social, basados en las redes) en la difusión de intervenciones de adaptación; los efectos no deseados sobre la justicia social y la integridad medioambiental de las estrategias de adaptación; las dificultades en la transmisión de conocimiento científico sobre adaptación a los responsables políticos, o la capacidad de las comunidades locales para implementar intervenciones de adaptación.

Esperamos que este artículo mejore la comprensión de los retos que enfrentan las ciudades de nuestro entorno en un escenario de cambio climático, al tiempo que contribuya a desarrollar la investigación social sobre las opciones y las intervenciones de adaptación más adecuadas en nuestras poblaciones. 


\section{Referencias bibliográficas}

AbHas, K. A.; Bloch, R. y Lamond, J. (2012). Cities and Flooding: A Guide to Integrated Urban Flood Risk Management for the 21st Century [en línea]. Washington: The World Bank. <http://gfdrr.org/gfdrr/node/1068>.

Abroms, L. C. y MaibaCH, E. W. (2008). "The Effectiveness of Mass Communication to Change Public Behavior». Annual Review of Public Health, 29, 219-234.

Adger, W. N.; Arnella, N. W. y Tompkins, E. L. (2005). «Successful adaptation to climate change across scales». Global Environmental Change, 15, 77-86.

AHERn, J. y GaLEA, S. (2011). "Collective Efficacy and Major Depression in Urban Neighborhoods». American Journal of Epidemiology, 173 (12), 1453-1462.

AKBARI, H. y Rose, L. S. (2001). Characterizing the fabric of the urban environment: A case study of metropolitan Chicago, Illinois. Berkeley, CA: Lawrence Berkeley National Laboratory Report LBNL-49275.

Akbari, H.; MenON, S. y Rosenfeld, A. (2009). "Global cooling: increasing solar reflectance of urban areas to offset CO2». Climatic Change, 95, 3-4.

Arnell, N. W. (2010). "Adapting to climate change: an evolving research programme». Climatic Change, 100, 107-111.

BACCINI, M. et al. (2008). "Heat effects on mortality in 15 European cities». Epidemiology, 19 (5), 711-9.

BALlESTER, F. (2005). «Contaminación atmosférica, cambio climático y salud». Revista Española de Salud Publica, 79 (2), 159-175.

Banco Mundial (2008). Climate Resilient Cities. Reducing Vulnerabilities to Climate Change Impacts and Strengthening Disaster Risk Management in East Asian Cities [en línea]. The International Bank for Reconstruction and Development / The World Bank. <www.worldbank.org/eap/climatecities>.

- (2011). Guide to Climate Change Adaptation in Cities [en línea]. The World Bank. <http://siteresources.worldbank.org/>.

Bauman, D. y Sims, J. H. (1978). «Flood Insurance: Some Determinants of Adoption». Economic Geography, 54 (3), 189-196.

Berrang-Ford, L.; Ford, J. D. y PATERSON, J. (2011). "Are we adapting to climate change?». Global Environmental Change, 21, 25-33.

Bierbaum, R. M. (2008). «Energy and Climate Change». Bulletin of the American Academy, 61 (2), 32-36.

Biesbroek, G. R.; Swart, R. J. y VAn Der KnAap, W. G. M. (2009). «The mitigationadaptation dichotomy and the role of spatial planning». Habitat International, 33, 230-237.

BURTON, I. (2005). «Adapt and thrive: options for reducing the climate change adaptation deficit». Policy Options, diciembre-enero, 32-38.

Callaway, J. M. (2004). «Adaptation benefits and costs: are they important in the global policy picture and how can we estimate them?». Global Environmental Change, 14, 273-282.

CISCAR, J. C. (ed.) (2009). Climate Change impacts in Europe: Final report of the PESE$T A$ research project [en línea]. JRC Scientific and Technical Report. <http://ftp.jrc. es/EURdoc/JRC55391.pdf>.

Clarck, A. (2009). Environmental Challenges to Urban Planning: Fringe areas, Ecological Footprints and Climate Change. Key Challenges in the Process of Urbanization in Ho Chi Minh City: Governance, Socio-Economic, and Environmental Issues Workshop. 
Climate Change Science Program (CCSP) (2003). Strategic Plan for the US Climate Change Science Program. A Report by the Climate Change Science Program and the Subcommittee on Global Change Research.

Climate InTERACTIVE [en línea]. <http://climateinteractive.org/>.

Comisión Europea (2009). Libro blanco. Adaptación al cambio climático: Hacia un marco europeo de actuación. Bruselas: Comisión Europea.

Dietz, T.; Ostrom, E. y STern, P. (2003). «The Struggle to Govern the Commons». Science, 302 (5652), 1907-1912.

Ebi, K. y SEmEnZA, J. (2008). "Community-Based Adaptation to the Health Impacts of Climate Change». American Journal of Preventive Medicine, 35 (5), 501-507.

Environmental Protection Agency (EPA). Reducing Urban Heat Islands: Compendium of Strategies Heat Island Reduction Activities [en línea]. <http://www.epa. gov/heatisld/resources/pdf/ActivitiesCompendium.pdf> [Consulta: marzo 2012].

Eriksen, S.; Aldunce, P. y Sekhar Bahinipati, C. et al. (2012). "When not every response to climate change is a good one: Identifying principles for sustainable adaptation». Climate and Development, 3 (1), 7-20.

FisCHER, E. M.; OlesOn, K. W. y LAWRENCE, D. M. (2012). «Contrasting urban rural heat stress responses to climate change». Geophysical Research Letters, 39.

Foster, J.; LOWE, A. y Winkelman, S. (2011). The value of green infrastructure for urban climate change adaptation [en línea]. Informe de The Center for Clean Air Policy. <www.ccap.org>.

Fouillet, A. et al. (2006). «Excess mortality related to the August 2003 heat wave in France». International Archives of Occupational and Environmental Health, 80, 16-24.

Füssel, H.-M. y Klein, R. J. T. (2006). «Climate Change Vulnerability Assessments: An Evolution of Conceptual Thinking». Climatic Change, 75(3), 301-329.

Giannakopoulos, C.; Bindi, M.; Moriondo, M.; Lesager, P. y Tin, T. (2005). Climate change impacts in the Mediterranean resulting from a $2 o$ C global temperature rise. World Wide Foundation.

GidDENS, A. (2010). La politica del cambio climático. Madrid: Alianza.

Glick, P.; Staudt, A. y Stein, B. (2009). A New Era for Conservation: Review of Climate Change Adaptation Literature. National Wildlife Federation Report.

HADDAD, B. (2005). «Ranking the adaptive capacity of nations to climate change when socio-political goals are explicit». Global Environmental Change, 15 (2), 165-176.

HAMin, E. y GuRRAN, N. (2009). «Urban form and climate change: Balancing adaptation and mitigation in the U.S. and Australia». Habitat International, 33, 238-245.

Heller, N. E. y Zavaleta, E. S. (2009). «Biodiversity management in the face of climate change: A review of 22 years of recommendations». Biological Conservation, $142,14-32$.

Houghton, J. T. Y.; Ding, D. J.; Griggs, M.; Noguer, P. J.; VAn Der Linden y Xiaosu, D. (eds.) (2001). Climate Change 2001: The Scientific Basis. Cambridge: Cambridge University Press.

International Housing Coalition (IHC) (2011). Adapting to Climate Change: Cities and the Urban Poor [en línea]. Washington: IHC. <http://intlhc.org/wp-content/uploads/2011/09/Climate-Change-and-the-Urban-Poor.pdf $>$.

Jacobson, M. Z. y Ten Hoeve, J. E. (2012). «Effects of Urban Surfaces and White Roofs on Global and Regional Climate». Journal of Climate, 25, 1028-1044.

KuinenberG, E. (2002). Heat Wave: A Social Autopsy of Disaster in Chicago. Chicago: University of Chicago Press. 
Kovats, R. S. y Hajat, S. (2008). «Heat Stress and Public Health: A Critical Review». Annual Review of Public Health, 29, 41-55.

Laukkonen, J.; Kim Blanco, P.; Lenhart, J.; Keiner, M.; Cavric, B. y KinuthIA-NJENGA, C. (2009). "Combining climate change adaptation and mitigation measures at the local level». Habitat International, 33, 287-292.

Lerch, D. (2007). Post Carbon Cities: Planning for Energy and Climate Uncertainty. Informe del Post Carbon Institute.

LOMborg, B. (2008). En frio: Guía del ecologista escéptico para el cambio climático. Madrid: Espasa-Calpe.

Lowe, A.; Foster, J. y Winkelman, S. (2009). Ask the Climate Question: Adapting to Climate Change Impacts in Urban Regions. Informe del Center For Clean Air Policy.

Machado, H. (2009). "Technological Challenges to Climate Change Adaptation and Negotiating Issues for Latin America». Comunicación presentada en el Latin American Regional Dialogue on Trade and Climate Change.

Mcmichael, A. J. y Butler, C. D. (2009). "Climate change and human health: recognising the really inconvenient truth». Medical Journal of Australia, vol. 191, 595-596.

Mcmichael, A. J.; Woodruff, R. E. y Hale, S. (2006). "Climate change and human health: present and future risks». The Lancet, 367 (9513), 859-69.

National Oceanographic and Atmospheric Administration (NOAA). Climate Program OfFICE (2011). Regional Integrated Sciences and Assessments (RISA) program [en línea]. Annual Report. <http://ine.uaf.edu/accap/documents/RISA_2011. pdf>.

Nordhaus, W. (2007). «A Review of the Stern Review on the Economics of Climate Change». Journal of Economic Literature, vol. XLV, 686-702.

O'BRIEN, K. (2009). "Climate change and values: do changing values define the limits to successful adaptation?». En: Adger W. N.; LorenZONI, I. y O’Brien, K. (eds.). Adapting to climate change: thresholds, values, governance. Cambridge: Cambridge University Press.

OKE, T. R. (1982). "The energetic basis of the urban heat island». Quarterly Journal of the Royal Meteorological Society, 108 (455), 1-24.

OlsEn, R. (2011). Climate Change and Risk-Informed Decision Making. Workshop on Scaling up World Bank's role in Disaster Risk Reduction [en línea]. Washington: Urban Flood Risk Management. <http://siteresources.worldbank.org/INTEAPREGTOPHAZRISKMGMT/Resources/4077899-1228926673636/4-Rolf_Olsen. pdf> [Consulta: marzo 2011].

Pardo, M. (2007). «El impacto social del cambio climático». Panorama Social, 5, 22-35.

PIZARRO, R. (2009). «The mitigation/adaptation conundrum in planning for climate change and human settlements: Introduction». Habitat International, 33, 227- 229.

Richardson, K.; StefFen, W.; Schellnhuber, H. J. et al. (2009). Climate Change: Global risks, Challenges and Decisions. Synthesis Report from the Congress.

Rifkin, J. (2011). The Third Industrial Revolution: How Lateral Power Is Transforming Energy, the Economy, and the World. Palgrave MacMillan.

Rojas BLANCO, A. (2006). "Local initiatives and adaptation to climate change». Disasters, 30 (1), 140-147.

RosenzWeIG et al. (2011). Responding to Climate Change in New York State. Synthesis Report. New York State Energy Research and Development Authority. 
SÁNCHEZ-RodrígueZ, R. (2009). "Learning to adapt to climate change in urban areas: A review of recent contributions». Current Opinion in Environmental Sustainability, 1, 201-206.

Satterthwaite, D.; HuQ, S.; Pelling, M.; Reid, H. y Romero LankaO, P. (2007). Adapting to Climate Change in Urban Areas: The possibilities and constraints in lowand middle-income nations. International Institute for Environment and Development (IIED). Human Settlements Discussion Paper Series.

Schernewski, G.; Hofstede, J. y Neumann, T. (eds.) (2011). Global Change and Baltic Coastal Zones. Coastal Research Library-Series. Springer, Dordrecht.

Semenza, J. C.; Rubin, Ch.; Falter, KH. et al. (1996). «Heat-related deaths during the July 1995 heat wave in Chicago». New England Journal of Medicine, 335 (2), 84-90.

Simon, F.; López-Abente, G.; Ballester, E. y Martínez, F. (2005). «Mortality in Spain during the heat waves of summer 2003». Euro Surveillance, 10 (7).

SMIT, B. y WANDEL, J. (2006). "Adaptation, adaptive capacity and vulnerability». Global Environmental Change, 16, 282-292.

Smith, P. y Olesen, J. E. (2010). «Synergies between the mitigation of, and adaptation to, climate change in agriculture». Journal of Agricultural Science, 148 (5), 543-552.

SPUR (2011). Climate Change hits home: Adaptation Strategies for the San Francisco Bay Area [en línea]. SPUR Report 05/2011. <http://www.spur.org/publications/ library/report/climate-change-hits-homes.

STERN, N. (2006). Stern Review on the Economics of Climate Change. Londres: HM Tresury.

Tierney, K.; Lindell, M. y PERRY, R. (2001). Facing the unexpected: disaster preparedness and response in the United States. Washington: Joseph Henry Press.

UNFCCC (2007). Investment and Financial Flows to Address Climate Change. Bonn: Climate Change Secretariat.

Wilson, CH. y Dowlatabadi, H. (2007). «Models of Decision Making and Residential Energy Use». Annual Review of Environmental Resources, 32,169-203.

WORLD METEREOLOGICAL ORGANIZATION (WMO) (2008). Urban flood risk management [en línea]. APFM Technical Document, 11. Flood Management Tools Series. $<$ http://www.apfm.info/pdf/ifm_tools/Tools_Urban_Flood_Risk_Management. $\mathrm{pdf}>$.

Yohe, G. y Tol, R. S. J. (2002). «Indicators for social and economic coping capacity-moving toward a working definition of adaptive capacity». Global Environmental Change, 12, 25-40. 